\title{
Traumatic versus non-traumatic spinal cord injuries: are there differential rehabilitation outcomes?
}

\author{
P Kennedy ${ }^{1,2}$ and ZJ Chessell ${ }^{2}$
}

Study design: Retrospective case review.

Objectives: To compare the rehabilitation outcomes between patients with traumatic spinal cord injuries and patients with nontraumatic spinal cord injuries, using The Needs Assessment Checklist (NAC), to observe if both the groups benefit from the same rehabilitation programme.

Setting: Tertiary care, spinal cord injury rehabilitation unit (National Spinal Injuries Centre), Stoke Mandeville Hospital, UK. Methods: Information obtained with the NAC at two time points during patients' rehabilitation was examined. Statistical analysis investigated the rehabilitation outcomes both between and within groups.

Results: Initial differences were observed on admission between patients with traumatic and non-traumatic spinal cord injuries in five of the ten rehabilitation domains measured, with patients with non-traumatic injuries presenting with better outcomes. At a later stage in patients' rehabilitation, however, differences between the groups had lessened. Furthermore, the results demonstrated that the whole cohort made significant improvements in all the ten rehabilitation domains, with the same finding evident for both patients with traumatic and patients with non-traumatic spinal cord injuries.

Conclusion: Patients with traumatic spinal cord injuries and patients with non-traumatic spinal cord injuries benefitted from the same rehabilitation programme in a spinal injury centre, making significant improvements in all ten rehabilitation domains measured, suggesting that it is effective to admit and rehabilitate patients with injuries resulting from both traumatic and non-traumatic aetiologies in the same specialised setting.

Spinal Cord (2013) 51, 579-583; doi:10.1038/sc.2013.27; published online 16 April 2013

Keywords: spinal cord injury; traumatic; non-traumatic; rehabilitation outcomes; The Needs Assessment Checklist

\section{INTRODUCTION}

An extensive worldwide literature survey found the incidence of spinal cord injury (SCI) to lie between 10.4 and 83 per million people per year, ${ }^{1}$ with a recent review demonstrating the crude incidence rates of traumatic SCI (T-SCI) to be between 12.1 per million in the Netherlands and 57.8 per million in Portugal, and non-traumatic SCI (NT-SCI) to lie at around 26.3 per million in Australia. ${ }^{2}$ Owing to the extensive lifelong consequences for the individual and the economic cost associated with SCI, ${ }^{1}$ resulting from both traumatic and nontraumatic aetiologies, research into the rehabilitation outcomes of these patients is essential. The demographic characteristics and rehabilitation outcomes of patients with T-SCI have been extensively studied, 3,4 whereas research into patients with NT-SCI has been noticeably limited until more recently, ${ }^{5,6}$ despite some studies suggesting the incidence of NT-SCI to be even greater than that of T-SCI. 3,7

Previous research comparing T-SCI and NT-SCI patients has demonstrated clear differences in demographic characteristics, ${ }^{8}$ together with similarities and differences in rehabilitation outcomes. Generally, studies have shown that NT-SCI patients had a significantly shorter length of stay in hospital, while T-SCI patients demonstrated significantly greater Functional Independence Measure $(\mathrm{FIM})^{9}$ change scores during rehabilitation. ${ }^{10,11}$ Recent research has found that when controlling for neurological deficits functional improvement was higher in patients with traumatic injuries, although the researchers suggested that this finding may be due to the younger age of these patients. ${ }^{12}$ Some studies have reported FIM admission scores to be significantly greater in patients with NT-SCI than T-SCI, ${ }^{10}$ although other studies have found no difference. ${ }^{11}$ Importantly, however, both patients with traumatic and nontraumatic injuries have demonstrated significant FIM change from admission to discharge, highlighting the effective rehabilitation of both the groups undergoing the same rehabilitation programme. ${ }^{10}$ Recent studies have found less pronounced differences in outcomes between the groups, albeit using different measures, demonstrating no significant differences in length of stay or functional outcomes, measured with The Barthel Index. ${ }^{13,14}$ Furthermore, a multi-centre study utilising the authors' own scale of measurement reported the rehabilitation of patients with NT-SCI to be as effective as that of T-SCI patients. ${ }^{15}$ Overall, this research appears to suggest that although patients with traumatic injuries may have greater functional improvement in rehabilitation overall, both the groups benefit significantly from similar rehabilitation programmes.

Rehabilitation for individuals with SCI needs to address more than just functional outcomes as patients' psychological well-being is also of paramount importance, both in regards to the close link to functional outcomes and in isolation. Overall, research has found the aetiology of SCI to have little or no effect on individuals' psychological well-being, in relation to anxiety, depression, stress and quality

${ }^{1}$ Oxford Institute of Clinical Psychology Training, University of Oxford, Oxford, UK and ${ }^{2}$ Department of Clinical Psychology, National Spinal Injuries Centre, Stoke Mandeville Hospital, Aylesbury, Buckinghamshire, UK

Correspondence: Professor P Kennedy, Department of Clinical Psychology, National Spinal Injuries Centre, Stoke Mandeville Hospital, Mandeville Road, Aylesbury, Buckinghamshire HP21 8AL, UK.

E-mail: paul.kennedy@hmc.ox.ac.uk

Received 7 February 2013; revised 12 March 2013; accepted 17 March 2013; published online 16 April 2013 
of life, ${ }^{16,17}$ and researchers have even suggested no apparent differences in post-traumatic stress disorder. ${ }^{18}$ These results, therefore, suggest that a similar approach to aiding patients' psychological wellbeing in rehabilitation settings would be effective in both individuals with traumatic and non-traumatic injuries.

A debate currently exists in some health services regarding whether patients with NT-SCI should be admitted and treated in the same settings as patients with T-SCI. Indeed, NT-SCI patients do not always receive rehabilitation in specialist spinal injury centres (SICs), resulting in rehabilitation conducted in a variety of settings, often creating uncoordinated care, lacking integration., ${ }^{79}$ It has been suggested that patients who complete their rehabilitation in nonspecialist centres, such as orthopaedic or general rehabilitation settings, have inferior outcomes to those managed in SICs, ${ }^{20}$ perhaps due to potentially insufficient resources to give patients the highly specialised care, information, therapy and equipment required for effective rehabilitation. ${ }^{19}$ The first study to examine patient outcomes in varied rehabilitation settings in the UK found that individuals who completed their rehabilitation at a SIC, as opposed to a non-spinal setting, presented with significantly better results in 10 out of 18 health outcomes, 16 out of 18 functional outcomes and 5 out of 10 social outcomes. ${ }^{20}$ Recent work by New et al. ${ }^{21}$ found that patients with non-traumatic injuries were less likely to be admitted to a SIC than those with traumatic injuries. Importantly, patients with non-traumatic injuries who were admitted and treated in a SIC had greater functional gains during rehabilitation than those who were not, a finding that interestingly was not evident in patients with traumatic injuries. It has, therefore, been recommended that patients with NT-SCI should receive rehabilitation in a SIC or a specialised neurological rehabilitation team. ${ }^{19-21}$

The majority of studies examining the rehabilitation outcomes of NT-SCI patients have utilised only length of stay, discharge destination and FIM scores as measurement, ${ }^{22}$ and, therefore, there is a need to examine in greater detail the full range of rehabilitation outcomes. The Needs Assessment Checklist (NAC) ${ }^{23}$ is a clinical assessment tool that comprehensively measures patients' needs, abilities and rehabilitation outcomes. The NAC adopts a biopsychosocial framework, measuring ten rehabilitation domains: physical health-care (PHC), activities of daily living $(\mathrm{ADL})$, skin and posture management, bladder management, bowel management, mobility, wheelchair and equipment (W\&E), community preparation (CP), psychological issues and discharge coordination (DC). Each domain is divided into three levels: the domain (that is, skin), the goal (that is, to be physically independent in performing skin checks) and the specific target (that is, knowledge of where and what to look for). Research has demonstrated the NAC to be a psychometrically reliable and valid assessment tool ${ }^{24,25}$ and a recent review found it to be one of the best measures of outcomes in SCI rehabilitation. ${ }^{26}$

This study will observe and compare rehabilitation outcomes, in the ten aforementioned domains of the NAC, between patients with T-SCI and NT-SCI undergoing the same rehabilitation programme at a SIC. This study aims to draw conclusions regarding whether NT-SCI patients benefit from the same rehabilitation programme as T-SCI patients and, therefore, whether it is advisable for them to be admitted and treated similarly.

\section{MATERIALS AND METHODS}

\section{Participants}

Unmatched sample. This study examined routinely collected data from individuals first admitted to The National Spinal Injuries Centre, Stoke Mandeville Hospital, UK, between February 2008 and November 2012.
Patients who had been previously admitted to The National Spinal Injuries Centre, who had not completed a NACl or a NAC2, and those with no information regarding their injury aetiology or date of admission were excluded from the analysis. Participants, therefore, consisted of 536 patients; 403 males (75\%) and 133 females (25\%), with American Spinal Injury Association Impairment Scale (AIS) grades of 200 AIS A (37\%), 59 AIS B (11\%), 117 AIS C (22\%), 139 AIS D (26\%) and 21 missing this information $(4 \%)$. The mean age at injury was 47.17 (median $=47.29$, s.d. $=18.16$, range $=0-88$ ), excluding eight patients missing this information, and the age range was $17-92$. The sample consisted of 346 patients with traumatic injuries $(65 \%)$ and 190 with non-traumatic injuries (35\%).

Matched sample. From the previous unmatched sample it was attempted to match all NT-SCI patients with a T-SCI patient, with both having completed a $\mathrm{NAC1}$ and NAC2 at the time of analysis. Criteria for matching consisted of gender, AIS grade (A, B, C or D) and the age at which their injury occurred (within five years), of which all three criteria were met. There were 21 NT-SCI patients for whom a T-SCI patient match could not be found and were, therefore, excluded from the analysis, as were those with no information regarding their AIS grade $(n=10)$ or age at injury $(n=4)$. This resulted in 144 patients; 72 with T-SCI and 72 with NT-SCI, 99 males (69\%) and 45 females (31\%), with 61 AIS A (42\%), 8 AIS B (6\%), 46 AIS C (32\%), 29 AIS D $(20 \%)$. The mean age at injury was $52.24($ median $=54.79$, s.d. $=13.34$, range $=20-76)$ and the age range was 23-79.

\section{Measurement}

The NAC, ${ }^{23}$ a clinical tool to assess rehabilitation outcomes, was utilised in this study. The ten domains of the NAC are scored on a four point scale $(0=$ completely dependent; $1=$ mostly dependent; $2=$ moderately independent; or $3=$ completely independent). Outcomes measured by $\mathrm{NAC} 1$ and NAC2 were examined in this study. The NAC1 is administered within 2 weeks of the patient mobilising and the NAC2 when the patient moves to a pre-discharge ward or 6 weeks before discharge. It should be noted that patients are able to reach full independence, despite injury severity, as the NAC can be scored in relation to physical or verbal independence. Item scores for each NAC subscale are totalled and a percentage of 'goals achieved' calculated and it is these ten percentage scores for each patient that are utilised in the analysis.

\section{Data analysis}

The data was analysed using the SPSS statistics version 17.0 (Chicago, IL, USA). A Shapiro-Wilk test was conducted initially to determine the likelihood that the data came from a normal distribution, which revealed that the majority of the data violated the assumption of normality. Exploratory MannWhitney tests were run on the unmatched sample to compare the ten domains of NAC1 rehabilitation outcomes between the two groups (T-SCI and NT-SCI) and subsequently to compare the same ten rehabilitation outcome domains from the NAC2 between the two groups. Wilcoxon signed-rank tests were then run on the sample matched for gender, AIS grade and age at injury, to examine the differences between the ten rehabilitation domains from NAC1 to NAC2 in the whole cohort, the T-SCI group and the NT-SCI group, while controlling for these factors.

\section{Statement of ethics}

We certify that all applicable institutional and governmental regulations concerning the ethical use of human volunteers were followed during the course of this research.

\section{RESULTS}

\section{Unmatched sample}

Differences in NAC1 outcomes between patients with T-SCI and NT-SCI. The differences between the ten (PHC, ADL, skin and posture management, bladder management, bowel management, mobility, W\&E, CP, psychological issues and DC) NAC1 outcomes were compared between patients with T-SCI and NT-SCI, with the 


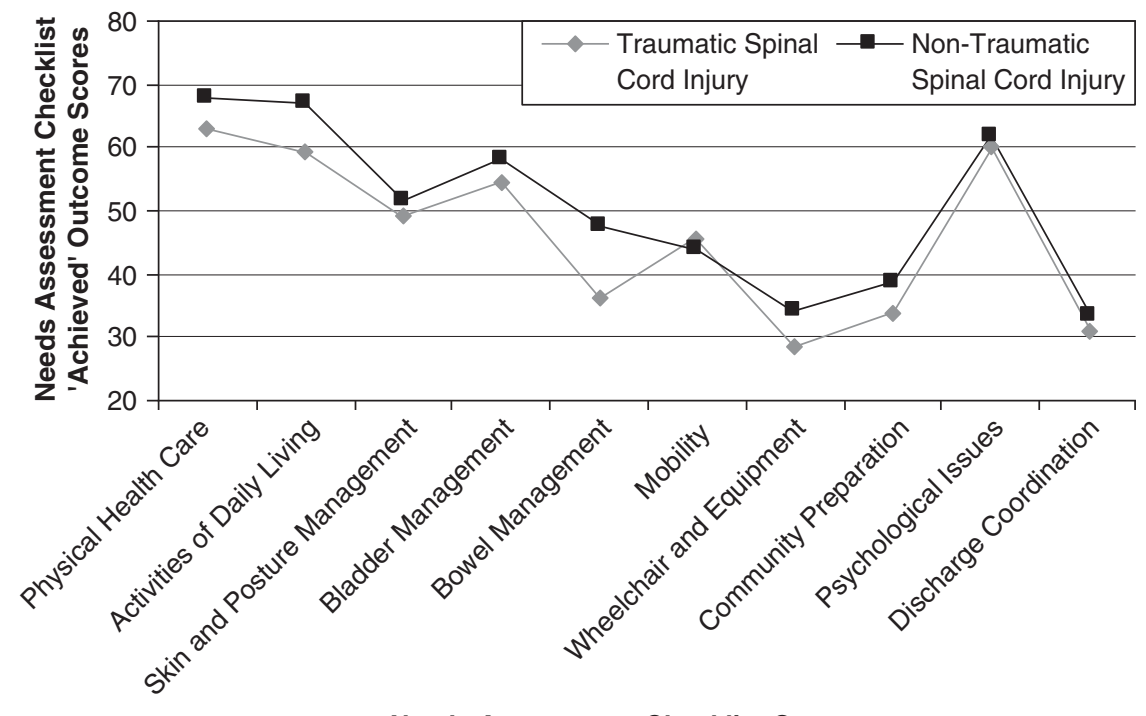

Needs Assessment Checklist Outcomes

Figure 1 Mean outcome scores of NAC one for patients with T-SCI and patients with non-T-SCI-Unmatched sample.

means displayed in Figure 1. A Mann-Whitney test was run on each outcome measure, which demonstrated significant differences between the groups in the outcomes of: PHC $(U=25263.0$, $P<0.01)$, ADL $(U=25631.0, \quad P<0.01)$, bowel management $(U=24469.5, \quad P<0.01)$, W\&E $(U=25939.0, \quad P<0.01)$ and $\mathrm{CP}$ $(U=25177.5, P<0.01)$, with NT-SCI patients demonstrating better outcomes at the time of NAC1.

Differences in NAC2 outcomes between patients with T-SCI and NT-SCI. Similarly, the differences between the ten NAC2 outcomes were compared between patients with T-SCI and NT-SCI, with the means displayed in Figure 2. A Mann-Whitney test was run on each outcome measure, which demonstrated significant differences between the groups in the outcomes of: $\operatorname{ADL}(U=10999.0, P<0.01)$ and mobility $(U=11502.5, P<0.05)$, with patients with T-SCI presenting with better outcome scores in mobility and patients with NT-SCI presenting with better outcome scores in ADL, at the time of NAC2.

\section{Matched sample}

Differences between NAC1 and NAC2 outcomes-Whole cohort. Analysis was then conducted using the sample with patients with T-SCI and NT-SCI matched for gender, AIS grade and age at injury, to examine the differences between NAC1 and NAC2 outcome scores with these factors controlled for, with the means displayed in Figure 3. A Wilcoxon signed-rank test was run on each outcome measure, with both T-SCI and NT-SCI groups included, which demonstrated significant gains in every outcome measure: $\mathrm{PHC}(Z=-9.926, P<0.01)$, ADL $(Z=-9.725, P<0.01)$, skin and posture management $(Z=-10.287$, $P<0.01)$, bladder management $(Z=-10.263, P<0.01)$, bowel management $(Z=-10.270, P<0.01)$, mobility $(Z=-9.821, P<0.01)$, W\&E $(Z=-10.382, P<0.01)$, CP $(Z=-10.346, P<0.01)$, psychological issues $(Z=-9.283, P<0.01)$ and DC $(Z=-10.236, P<0.01)$. These results clearly demonstrate rehabilitation to be effective overall.

Differences between NAC1 and NAC2 outcomes-Patients with T-SCI and NT-SCI. The same matched sample was then used to examine the differences between NAC1 and NAC2 outcome scores for patients with T-SCI and patients with NT-SCI separately. A Wilcoxon signed-rank test was run on each outcome measure in each group, which demonstrated significant gains in all rehabilitation domains in both the groups.

In regards to patients with T-SCI, significant differences between NAC1 and NAC2 were found in all outcomes: PHC $(Z=-7.030$, $P<0.01)$, ADL $(Z=-6.864, P<0.01)$, skin and posture management $(Z=-7.374, P<0.01)$, bladder management $(Z=-7.300, P<0.01)$, bowel management $(Z=-7.324, P<0.01)$, mobility $(Z=-7.004$, $P<0.01)$, W\&E $(Z=-7.369, P<0.01), \mathrm{CP}(Z=-7.276, P<0.01)$, psychological issues $(Z=-7.064, P<0.01)$ and DC $(Z=-7.308$, $P<0.01)$.

Similarly, patients with NT-SCI presented with significant differences between NAC1 and NAC2 in all domains: PHC $(Z=-7.045$, $P<0.01)$, ADL $(Z=-6.921, P<0.01)$, skin and posture management $(Z=-7.171, P<0.01)$, bladder management $(Z=-7.215, P<0.01)$, bowel management $(Z=-7.214, P<0.01)$, mobility $(Z=-6.902$, $P<0.01)$, W\&E $(Z=-7.344, P<0.01), \mathrm{CP}(Z=-7.375, P<0.01)$, psychological issues $(Z=-6.020, P<0.01)$ and DC $(Z=-7.188$, $P<0.01)$.

\section{DISCUSSION}

Previous literature has highlighted the importance of investigating the rehabilitation outcomes of patients with NT-SCI. Firstly, this is necessary to increase knowledge, as there is a current paucity of studies in the area, in comparison with extensive research examining the outcomes of patients with T-SCI. This is particularly essential as the aging population has resulted, and will continue to result in, large increases in NT-SCI. ${ }^{7,19}$ Secondly, research will help bring clarification to the debate regarding the setting in which NT-SCI patients should be rehabilitated, important for health-care guidelines and recommendations.

The results from NAC1 demonstrated initial differences between the groups in five of the ten rehabilitation domains measured, with NT-SCI patients presenting with better outcomes in PHC, ADL, bowel management, $\mathrm{W} \& \mathrm{E}$ and $\mathrm{CP}$. Indeed, previous work has demonstrated NT-SCI patients to have less clinically severe injuries ${ }^{15}$ and greater FIM admission scores, ${ }^{10}$ perhaps suggesting that NT-SCI patients begin rehabilitation at a more advanced level than those with T-SCI. However, the results showed that as rehabilitation progressed 


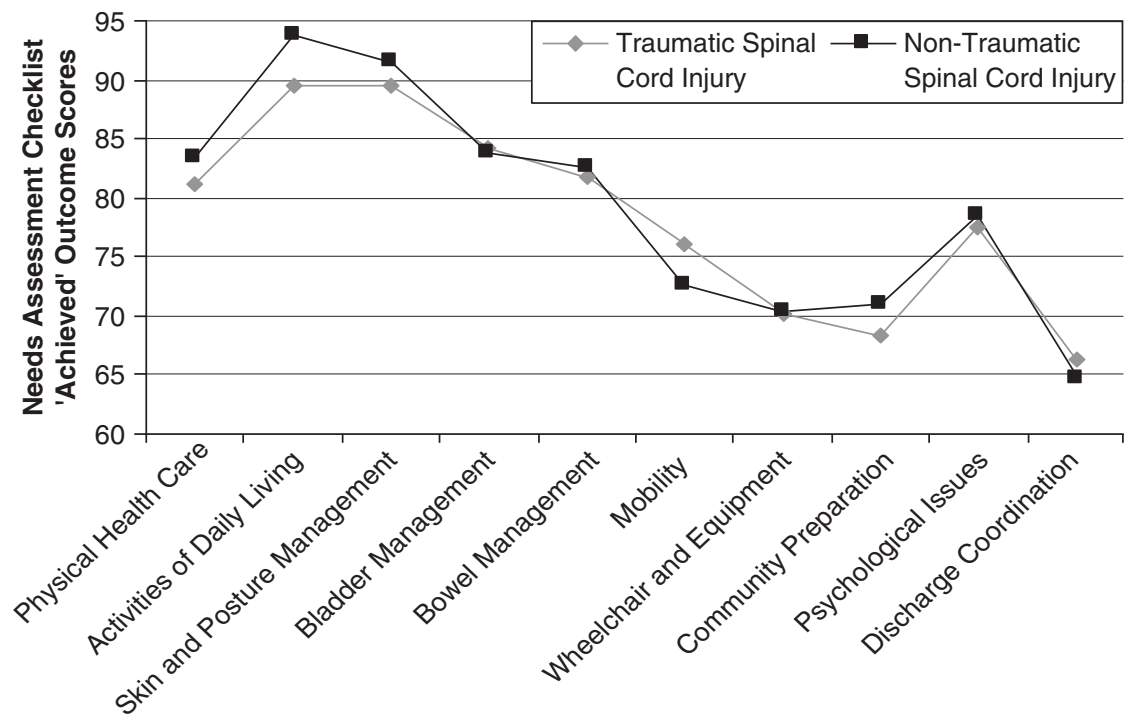

Needs Assessment Checklist Outcomes

Figure 2 Mean outcome scores of NAC two for patients with T-SCl and patients with non-T-SCl—Unmatched sample.

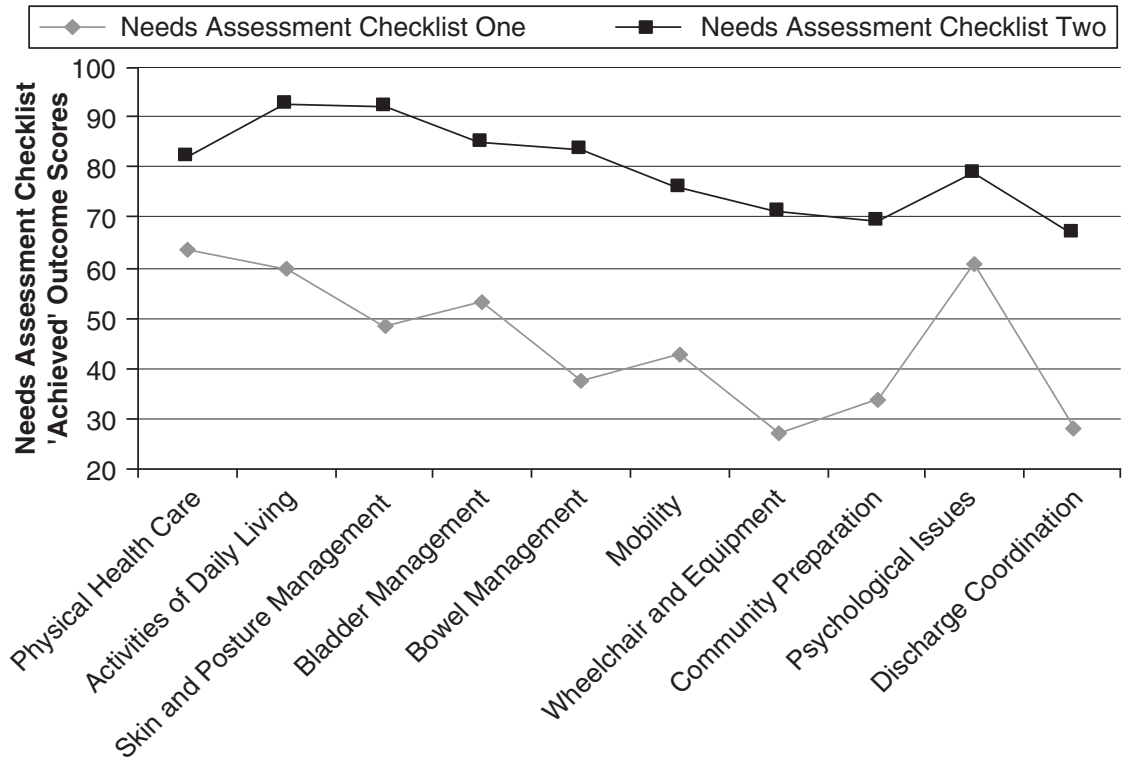

Needs Assessment Checklist Outcomes

Figure 3 Mean outcome scores of NAC one and NAC two for the whole cohort-Matched sample.

differences between the groups lessened, with NT-SCI patients presenting with better scores in the domain of ADL at the time of NAC2, and T-SCI patients presenting with significantly better outcome scores in the mobility domain. It should be noted that when this analysis was run with the smaller sample matched for gender, AIS grade and age at injury, fewer differences were evident between the groups: at NAC1 NT-SCI patients had better scores in PHC and ADL and at NAC2 NT-SCI patients had better scores in ADL, supporting the idea that patients with traumatic and patients with non-traumatic injuries demonstrate similarities throughout their rehabilitation. Indeed, recent research has demonstrated T-SCI patients to have lower autonomy in daily living activities on admission, as measured by the Barthel Index for activities of daily life independence, ${ }^{13}$ which the authors suggested could be related to
T-SCI patients' associated lesions. Importantly, however, these researchers also found that patients' injury aetiology did not affect their rehabilitation prognosis, as individuals with traumatic and individuals with non-traumatic injuries achieved similar neurological and functional results at discharge. ${ }^{27}$ This research found, therefore, that although patients with T-SCI achieved greater improvement in Barthel Index score throughout rehabilitation, similarities were evident between the groups in the end result of rehabilitation.

Despite these initial differences it is clear that both T-SCI and NT-SCI patients benefitted from the same rehabilitation programme at a SIC, with significant improvements between NAC1 and NAC2 found in all ten rehabilitation domains in both the groups, with all improvements significant at the $P<0.01$ level. These findings suggest 
that the needs of both NT-SCI and T-SCI patients are targeted with the same rehabilitation programme and, therefore, it appears advisable that NT-SCI patients undergo rehabilitation in the same settings as T-SCI patients. Indeed, previously mentioned work has shown that both T-SCI and NT-SCI patients benefitted from rehabilitation in the same settings, ${ }^{15}$ had significant FIM changes from admission to discharge, ${ }^{10}$ and demonstrated no difference in length of stay or functional outcomes. ${ }^{14}$ The first study to conduct regression analysis to examine the influence of injury aetiology on functional outcomes found it not to be an independent determinant. These researchers suggested that the bivariate association of aetiology with functional outcome found could be due to differences between T-SCI and NT-SCI patients' age, lesion characteristics and/or functionality at admission. ${ }^{15}$ The current work adopts a comprehensive measure of rehabilitation yet to be utilised to examine the differences between the outcomes of patients with traumatic and patients with non-traumatic injuries, with the results clearly supporting research that has shown similarities in rehabilitation outcomes between these groups.

As the scope of this research does not extend to a comparison of rehabilitation outcomes of SCI patients in a non-spinal setting direct conclusions cannot be drawn regarding the advantage of rehabilitation for T-SCI and NT-SCI patients in a specialised setting. However, much previous work has suggested this to be the case. Early work stated that SICs were established due to 'poor results obtained when SCI patients were managed sporadically in small numbers in nonspecialised departments (p. 239), ${ }^{28}$ and, therefore, it is well accepted that T-SCI patients should be treated in specialised settings. Patients with non-traumatic injuries, however, often receive rehabilitation in non-specialised settings despite a wealth of research suggesting that both the groups should be rehabilitated in a SIC or a specialised neurological rehabilitation team. ${ }^{19-21}$ It has been stated that 'patients not cared for in specialised units may have worse outcomes, such as greater disability at discharge, longer hospital admission, higher rates of discharge to nursing homes and more preventable complications (p. 34). ${ }^{29}$ Furthermore, a survey of physicians found that $85 \%$ believed NT-SCI patients should be rehabilitated in an SIC or a neurological rehabilitation unit specialising in NT-SCI. ${ }^{19}$ An editorial in Spinal Cord stated that NT-SCI patients should have equal access to the care provided by SICs and made the valid point that a matched-case-control study to demonstrate this would not be ethical. ${ }^{30}$

In conclusion, despite initial differences, with NT-SCI patients presenting with better outcome scores in certain rehabilitation domains on admission, both groups made significant improvements during a rehabilitation programme at a SIC. This suggests that patients with T-SCI and patients with NT-SCI should, therefore, be admitted and treated in the same rehabilitation setting.

\section{DATA ARCHIVING}

There were no data to deposit.

\section{CONFLICT OF INTEREST}

The authors declare no conflict of interest.

\section{ACKNOWLEDGEMENTS}

We would like to thanks all patients and staff at The National Spinal Injuries Centre, Stoke Mandeville Hospital who participated in this clinical audit.
1 Wyndaele M, Wyndaele J-J. Incidence, prevalence and epidemiology of spinal cord injury: what learns a worldwide literature survey? Spinal Cord 2006; 44: 523-529.

2 van den Berg MEL, Castellote JM, Mahillo-Fernandez I, de Pedro-Cuesta J. Incidence of spinal cord injury worldwide: a systematic review. Neuroepidemiology 2010; 34: 184-192.

3 DeVivo MJ. Epidemiology of traumatic spinal cord injury: trends and future implications. Spinal Cord 2012; 50: 365-372.

4 Eastwood EA, Hagglund KJ, Ragnarsson KT, Gordon WA, Marino RJ. Medical rehabilitation length of stay and outcomes for persons with traumatic spinal cord injury - 1990-1997. Arch Phys Med Rehabil 1999; 80: 1457-1463.

5 Ronen J, Goldin D, Bluvshtein V, Fishel B, Gelernter I, Catz A. Survival after nontraumatic spinal cord lesions in Israel. Arch Phys Med Rehabil 2004; 85: 1499-1502.

6 New PW, Cripps RA, Bonne Lee B. Global maps of non-traumatic spinal cord injury epidemiology: towards a living data repository. Spinal Cord 2013; doi:10.1038/ sc.2012.165).

7 New PW, Sundararajan V. Incidence of non-traumatic spinal cord injury in Victoria, Australia: a population-based study and literature review. Spinal Cord 2008; 46: 406-411.

8 New PW, Simmonds F, Stevermuer T. A population-based study comparing traumatic spinal cord injury and non-traumatic spinal cord injury using a national rehabilitation database. Spinal Cord 2011; 49: 397-403.

9 Guide for the Uniform Data Set for Medical Rehabilitation (Including the FIMTM Instrument), version 5.1. State University of New York at Buffalo: Buffalo, NY, Uniform Data Set for Medical Rehabilitation 1997.

10 McKinley WO, Seel RT, Hardman JT. Nontraumatic spinal cord injury: incidence, epidemiology, and functional outcome. Arch Phys Med Rehabil 1999; 80: 619-623.

11 McKinley WO, Seel RT, Gadi RK, Tewksbury MA. Nontraumatic vs. traumatic spinal cord injury: a rehabilitation outcome comparison. Am J Phys Med Rehabil 2001; 80: 693-699.

12 Aidinoff E, Benjamini Y, Galili T, Polliack T, Front L, Bluvshtein V et al. Non-linear formulas for the spinal cord injury ability realization measurement index. Spinal Cord 2012; 50: 324-327.

13 Mahoney FI, Barthel DW. Functional evaluation: The Barthel Index. Maryland State Med J 1965; 14: 61-65.

14 Gupta A, Taly AB, Srivastava A, Vishal S, Murali T. Traumatic vs non-traumatic spinal cord lesions: comparison of neurological and functional outcome after in-patient rehabilitation. Spinal Cord 2008; 46: 482-487.

15 Osterthun R, Post MWM, van Asbeck FWA. Characteristics, length of stay and functional outcome of patients with spinal cord injury in Dutch and Flemish rehabilitation centres. Spinal Cord 2009; 47: 339-344.

16 Migliorini CE, New PW, Tonge BJ. Comparison of depression, anxiety and stress in persons with traumatic and non-traumatic post-acute spinal cord injury. Spinal Cord 2009; 47: 783-788.

17 Migliorini CE, New PW, Tonge BJ. Quality of life in adults with spinal cord injury living in the community. Spinal Cord 2011; 49: 365-370.

18 Lude P, Kennedy P, Evans M, Lude Y, Beedie A. Post traumatic distress symptoms following spinal cord injury: a comparative review of European Samples. Spinal Cord 2005; 43: 102-108.

19 New PW. Non-traumatic spinal cord injury: what is the ideal setting for rehabilitation? Aust Health Rev 2006; 30: 353-361.

20 Smith M. Efficacy of specialist versus non-specialist management of spinal cord injury within the UK. Spinal Cord 2002; 40: 11-16.

21 New PW, Simmonds F, Stevermuer T. Comparison of patients managed in specialised spinal rehabilitation units with those managed in non-specialised rehabilitation units. Spinal Cord 2011; 49: 909-916.

22 New PW. Functional outcomes and disability after nontraumatic spinal cord injury rehabilitation: results from a retrospective study. Arch Phys Med Rehabil 2005; 86: 250-261.

23 Kennedy P, Hamilton LR. The needs assessment checklist: a clinical approach to measuring outcome. Spinal Cord 1999; 37: 136-139.

24 Berry C, Kennedy P. A psychometric analysis of the Needs Assessment Checklist (NAC). Spinal Cord 2002; 41: 490-501.

25 Kennedy P, Smithson EF, Blakey LC. Planning and structuring spinal cord injury rehabilitation: The Needs Assessment Checklist. Top Spinal Cord Inj Rehabil 2012; 18: 135-137.

26 Dawson J, Shamley D, Jamous MA. A structured review of outcome measures used for the assessment of rehabilitation interventions for spinal cord injury. Spinal Cord 2008; 46: 768-780.

27 Scivoletto G, Farchi S, Laurenza L, Molinari M. Traumatic and non-traumatic spinal cord lesions: an Italian comparison of neurological and functional outcomes. Spinal Cord 2011; 49: 391-396.

28 Frankel H. Spinal cord injury units. Paraplegia 1987; 25: 239-240.

29 New PW, Townson A, Scivoletto G, Post MWM, Eriks-Hoogland I, Gupta A et al. International comparison of the organisation of rehabilitation services and systems of care for patients with spinal cord injury. Spinal Cord 2012; 51: 33-39.

30 Silver JR. Medical cases who would benefit from treatment on a spinal injury unit. Spinal Cord 2004; 42: 435-437. 